\title{
Analytical Solution for Motion Around Radiated Varying Mass Body
}

\author{
M. A. Bin_Humaidan', M. I. El-Saftawy ${ }^{2}$ and H. M. Asiri ${ }^{3}$ \\ 1,2,3 Current Address: King Abdul-Aziz University, Faculty of Science, Department of Astronomy and Space, \\ Jeddah, K.S.A
}

E. Mail: marwahumaidan36@gmail.com

${ }^{2}$ Permanent Address: National Research Institute of Astronomy and Geophysics, Helwan, Cairo, Egypt.

malsaftawy@kau.edu.sa

\begin{abstract}
In this work we will add the radiation pressure effect of varying mass body to the model of varying mass Hamiltonian function, including Periastron effect. The problem was formulated in terms of Delaunay variables. The solution of the problem was constructed based on Delava - Hansilmair perturbation techniques. Finally we find the first order solution for the problem as time series by calculating the desired order for the D operator and variables..
\end{abstract}

Keywords : Varying Mass, Radiation Pressure,Perturbations Techniques

\section{1) Introduction}

The two-body problem with variable mass was considered mathematical problem since none of the physicists worked in it, until Jeans, 1924 [1] was the first to pose this as astrophysical problem basing his studies on the theories of Eddington on the relationship between luminosity and star mass.

Physically different cases of the problem of two bodies with variable masses are classified by Razbitnaya,[2] , where he listed twenty-two of Meshcherskii equation for two basic classes: the generalized two-body problem with both bodies moving in the inertial system, and the confined two-body problem, with the central body at rest in the inertial system. Their obtained when the masses vary according to the hypothesis of Mescherskii [3].

Berkovich $[4,5]$ has published several research papers on the properties of the Gylden-Meshherskii equations, and the feasibility of converting them into the form autonomous equations. He analyzed the integrable cases and established all possible laws of mass change under which the unperturbed Gylden-Meshcherskii problem is reducible to the autonomous form.

Salmassi [6], introduced paper to discuss the second order adiabatic invariants in the two-body problem with slowly varying mass. in his search, he used action and angle variables, adiabatic invariants to order one are found.

An ample list can be found in the published works of Polyakova [7] and Prieto [8]. The specific case which lead to slow isotropic mass loss, many researcher have been their focus like, for instance, Hadjidemetriou [9, 10], Van Der Laan and Verhulst [11], Verhulst [12, 13], Verhulst and Eckhaus [14]. The vast majority of these, in search of the stellar application, have taken the so-called Eddington-Jeans law, Jeans [15] as a law of the variation of mass.

$$
\dot{m}=-\alpha m^{n}
$$


where $\alpha$ and $n$ are real numbers, the first of them positive proximate to zero and $n$ varying between 1.4 and 4.4. So, for example, Hadjidemetriou used this to integrate the Lagrange equations, first numerically and then analytically, considering the problem as one of two-body perturbation.

Prieto \& Docobo [16, 17], found an approximate analytic solution of the two-body problem with slowly decreasing mass which is obtained through the integration of the Hamilton equations using Deprit's method of perturbations. The solution, resolved from the law of mass variation, Eqn. (1), is put into practice in a specific case and compared with Mestschersky's exact equation; $n=2$; and with that which results from numerically integrating the equations.

Considering this problem of celestial mechanics, it has been addressed by, Docobo, Blanco and Abelleira [18], Andrade and Docobo [19], and others.

In [20], Andrade analyzed the dynamics of binary systems with time dependent mass loss and periastron effect i.e., a supposed enhanced mass loss during periastron passage- by means of analytical and numerical techniques.

Andrade and Docobo [21] studied the dynamics of binary systems with small parameter perturbation model, the time-dependence of the whole set of orbital elements, concluded, could be calculated over long timescales and even for high eccentricities. In these models, they studied the following time- and distance-dependent mass-loss law:

$$
\dot{\mu}\left(t ; r ; P_{\theta}\right)=\dot{\mu}(t)-\beta \frac{P_{\theta}}{r^{2}}
$$

Where the first term represent time-dependent mass loss, and the last one introduces the periastron effect where " $r$ " is the distance between the two components, $P \theta$ is the total angular momentum and $\beta$ is another small parameter close to zero.

Rahoma et al., [22], presented a scientific paper about the two-body problem with varying mass in case of isotropic mass loss from both components of the binary systems. The law of mass variation used gives rise to a perturbed Keplerian problem depending on two small parameters. The problem is treated analytically in the Hamiltonian frame-work and the equations of motion are integrated using the Lie series developed and applied, separately by Delva [23] and Hanslmeier [24]. A second order theory of the two bodies eject mass is constructed, returning the terms of the rate of change of mass up to second order in the small parameters of the problem.

Amirah [25] , she worked on providing research for the two bodies with slowly varying mass, was obtained taking into consideration the periastron effect. The solution was obtained through constructing a second order canonical transformation using "Hori's" method developed by "Kamel". The elements of the transformation as well as the inverse transformation were obtained too. The final solution of the problem was derived using "DelvaHanslmeier" method. During the analysis, there was a secular perturbation in the argument of periastron due to the varying mass. The equation for the variable associated to the variable mass was derived. The equations for calculating the perturbed orbital. 


\section{2) Materials and Methods}

\section{I-The Hamiltonian of The Problem}

The Hamiltonian for the two-body problem expressed in term Delanuay variables, which derived firstly by Deprit, A.[26], is:

$$
H\left(q_{1} \cdot Q_{1} \cdot Q_{2} ; t\right)=-\frac{1}{2} \frac{\mu^{2}}{Q_{1}^{2}}+\frac{\dot{\mu}}{\mu} Q_{1} e \sin E
$$

where the usual Delaunay Variable defined by:

$q_{1}=$ Mean anamoly. $\quad q_{2}=\omega . \quad q_{3}=\Omega$

$$
Q_{1}=\sqrt{\mu a} . \quad Q_{2}=Q_{1} \sqrt{1-e^{2}} . \quad Q_{3}=Q_{2} \cos I
$$

$q_{i}^{\prime} s$ are considered as the coordinates, while $Q_{i}^{\prime} s$ are their corresponding conjugate momenta.

The variation of $\mu$ may be retained from one of the two masses $m_{-} 1$ or $m_{-} 2$, and this is the case of one body eject mass. We will concern with the first case.

The Hamiltonian $H$ represented by Eq. (3) is depending implicitly on time through the variable mass $\mu$ and its time derivative $\dot{\mu}$. By modifying Docobo's law for the rate of change of mass assigned by eq. (3) and use Jeans law described by Eq. (2), we get:

$$
\dot{\mu}(r, t, \mu)=-\alpha \mu^{n}-\beta \frac{Q_{2} \mu^{2}}{Q_{1}^{4}}\left(\frac{a}{r}\right)^{2}
$$

Substituting from Eq. (4) into Eq. (3) yields:

$$
H=-\frac{1}{2} \frac{\mu^{2}}{Q_{1}^{2}}+\frac{\dot{\mu}(t)}{\mu} Q_{1} e \sin E-\beta \frac{Q_{2} e \mu}{Q_{1}^{3}}\left(\frac{a}{r}\right)^{2} \sin E
$$

Since the variable mass, $\mu$, and its time derivatives can be expressed as a Taylor series expansion, then the Hamiltonian (5) can be expressed as:

$$
\begin{aligned}
& H=-\frac{\mu_{0}^{2}}{2 Q_{1}^{2}}+\frac{\alpha}{Q_{1}^{2}} \mu_{0}^{n+1}\left(t-t_{0}\right)-\frac{\alpha^{2}}{2 Q_{1}^{2}} \mu_{0}^{2 n}(n+1)\left(t-t_{0}\right)^{2}- \\
& \alpha Q_{1} e \mu_{0}^{n-1} \sin E+\alpha^{2} Q_{1} e \mu_{0}^{2}{ }^{n-2}(n-1)\left(t-t_{0}\right) \sin E- \\
& \beta \frac{Q_{2} e \mu_{0}}{Q_{1}^{3}}\left(\frac{a}{r}\right)^{2} \sin E+\beta \alpha \frac{Q_{2}}{Q_{1}^{3}} \text { e } \mu_{0}^{n}\left(t-t_{0}\right)\left(\frac{a}{r}\right)^{2} \sin E \\
& H=H=H_{0}+H_{\alpha}+H_{\beta}+H_{\alpha \beta}
\end{aligned}
$$

Where;

$$
\begin{aligned}
& H_{0}=-\frac{\mu_{0}^{2}}{2 Q_{1}^{2}} \\
& H_{\alpha}=\frac{\alpha}{Q_{1}^{2}} \mu_{0}^{n+1}\left(t-t_{0}\right)-\frac{\alpha^{2}}{2 Q_{1}^{2}} \mu_{0}^{2 n}(n+1)\left(t-t_{0}\right)^{2}-\alpha Q_{1} e \mu_{0}^{n-1} \sin E+\alpha^{2} Q_{1} e \mu_{0}^{2}{ }^{n-2}(n-1)\left(t-t_{0}\right) \sin E,
\end{aligned}
$$




$$
\begin{aligned}
& H_{\beta}=-\beta \frac{Q_{2}}{Q_{1}^{3}} \text { e } \mu_{0}\left(\frac{a}{r}\right)^{2} \sin E \\
& H_{\alpha \beta}=\beta \alpha \frac{Q_{2}}{Q_{1}^{3}} \text { e } \mu_{0}^{n}\left(t-t_{0}\right)\left(\frac{a}{r}\right)^{2} \sin E
\end{aligned}
$$

The potential due to radiation pressure $U_{R}$ can be derived assuming the conservative force relation:

$$
\mathrm{U}_{\mathrm{R}}=\frac{\Phi}{Q_{1}^{2}} \boldsymbol{R}(\gamma)\left[\mu_{0}-\alpha \mu_{0}^{n}\left(t-t_{0}\right)+\frac{1}{2 !} \alpha^{2} \mu_{0}^{2 n-1} n\left(t-t_{0}\right)^{2}\right]
$$

With, $\Phi=\frac{a}{r}$ and $R(\gamma)=\frac{A}{m} \frac{S_{0}}{c}(1+\alpha) r_{0}^{2} \cos ^{2} \gamma$

In last equation, $\frac{A}{m}, \mathrm{C}$, and $S_{0}$ are the area to mass ratio for the spacecraft, speed of light and the luminosity of the radiated object with radius $\mathrm{r}_{0}$ respectively. While $\alpha$ and $\gamma$ are the reflection coefficient of the spacecraft surface and the angle of incident of the radiation with the normal to the surface respectively.

After performing the Hamilton for the varying mass, represented by Eqn. (6), and the radiation pressure, represented by Eqn. (7), the final Hamilton function can written as:

$$
\begin{aligned}
& \mathcal{H}=\mathrm{T}+\mathrm{U} \\
& \mathcal{H}=\mathrm{H}_{0}+\mathrm{H}_{\alpha}+\mathrm{H}_{\beta}+\mathrm{H}_{\alpha \beta}+\mathrm{U}_{\mathrm{R}}
\end{aligned}
$$

\section{II-Method of Soluation}

There are several cases in celestial mechanics, the series development of the disturbing function is a difficult problem and complicated. To avoid this the procedure can be performed with an operator. A special linear differential operator, the Lie operator, produce a Lie series; The convergence of the Lie series is the same as a Taylor series, because it is considered another analytical from of a Taylor series.

- Delva-Hanslmeier Method

Consider the Hamiltonian, $\mathcal{H}(x, y, X, Y)$ is function of the angle variables, $X, Y$ and there conjugate momenta $x, y$. Then the equation of motion are:

$$
\begin{array}{cc}
\dot{x}=\frac{d x}{d t}=\frac{\partial \mathcal{H}}{\partial x} & X=\frac{d x}{d t}=-\frac{\partial \mathcal{H}}{\partial x} \\
\dot{y}=\frac{d y}{d t}=\frac{\partial \mathcal{H}}{\partial y .} & Y=\frac{d y}{d t}=-\frac{\partial \mathcal{H}}{\partial y}
\end{array}
$$

The linear Lie operator has the general form:

$$
D=\frac{\partial}{\partial x} \frac{d x}{d t}+\frac{\partial}{\partial y} \frac{d y}{d t}+\frac{\partial}{\partial x} \frac{d x}{d t}+\frac{\partial}{\partial y} \frac{d y}{d t}+\frac{\partial}{\partial t}
$$

The solution $\vec{x}(x, y, X, Y, t), \vec{y}(x, y, X, Y, t), \vec{X}(x, y, X, Y, t)$ and $\vec{Y}(x, y, X, Y, t)$ are then given by the Lie series: 


$$
\begin{gathered}
\vec{x}(x, y, X, Y, t)=\left[\left\{\exp \left(t-t_{0}\right) D\right\} x\right]_{\vec{x}=\vec{x}_{0}} \\
=\sum_{j=0}\left[D^{j} \vec{x}\right]_{\vec{x}_{0}} \frac{\left(t-t_{0}\right)^{j}}{j !} \\
\vec{y}(x, y, X, Y, t)=\left[\left\{\exp \left(t-t_{0}\right) D\right\} y\right]_{\vec{y}=\vec{y}_{0}} \\
=\sum_{j=0}\left[D^{j} \vec{y}\right]_{\vec{y}_{0}} \frac{\left(t-t_{0}\right)^{j}}{j !} \\
\vec{X}(x, y, X, Y, t)=\left[\left\{\exp \left(t-t_{0}\right) D\right\} X\right]_{\vec{X}=\vec{X}_{0}} \\
=\sum_{j=0}\left[D^{j} \vec{X}\right]_{\vec{X}_{0}} \frac{\left(t-t_{0}\right)^{j}}{j !} \\
\vec{Y}(x, y, X, Y, t)=\left[\left\{\exp \left(t-t_{0}\right) D\right\} Y\right]_{\vec{Y}=\vec{Y}_{0}} \\
=\sum_{j=0}\left[D^{j} \vec{Y}\right]_{\vec{Y}_{0}} \frac{\left(t-t_{0}\right)^{j}}{j !}
\end{gathered}
$$

where $D^{j} \vec{x}, D^{j} \vec{y}, D^{j} \vec{X}$, and $D^{j} \vec{Y}$ are to be evaluated for initial conditions

$\overrightarrow{x_{0}}\left(x_{0}, y_{0}, X_{0}, Y_{0}, t_{0}\right) \cdot \overrightarrow{y_{0}}\left(x_{0}, y_{0}, X_{0}, Y_{0}, t_{0}\right), \overrightarrow{X_{0}}\left(x_{0}, y_{0}, X_{0}, Y_{0}, t_{0}\right)$ and $\overrightarrow{Y_{0}}\left(x_{0}, y_{0}, X_{0}, Y_{0}, t_{0}\right)$

\section{III- Algorithms for Computation}

The first step

1- $\quad$ Formulate the Hamiltonian of the problem under concern, $\tilde{H}(x, y)$. Where $(x, y)$ are $2 \mathrm{n}$ vector.

2- Using the Hamilton canonical equations of motion to obtain the rate of change in the canonical elements:

$$
\frac{d x_{i}}{d t}=\frac{\partial \tilde{H}(x, y)}{\partial y_{i}} \text { and } \frac{d y_{i}}{d t}=-\frac{\partial \tilde{H}(x, y)}{\partial x_{i}}
$$

The second step

1- Constructing the Lie operator " $D$ " for the problem.

$$
D=\frac{d}{d t}=\sum_{i=1}^{n}\left(\frac{\partial}{\partial x_{i}} \frac{d x_{i}}{d t}+\frac{\partial}{\partial y_{i}} \frac{d y_{i}}{d t}\right)+\frac{\partial}{\partial t}
$$

2- $\quad$ Constructing the solution of the problem in the form of Lie series 


$$
\begin{aligned}
\vec{S}\left(x_{i}, y_{i}\right) & =\left[\exp \left(t-t_{o}\right) D \vec{S}\right]_{\vec{S}=\vec{S}_{o}} \\
& =\sum_{J=0}^{\infty}\left[D^{j} \vec{S}\right]_{\vec{S}_{o}} \frac{\left(t-t_{o}\right)^{j}}{j !}
\end{aligned}
$$

where $\vec{S}$ is any one of the canonical variable and $\vec{S}_{0}$ its value at time to.

The third step

Apply the above two steps into the system of equation to get the solution for the problem.

\section{3) Results and Discussion}

In this part we will apply the steps of the Delva-Hanselmier method, by finding the equation of motion for problem under concern. The solution will be evaluated during the steps was described in the last part.

\section{I- Development of the Hamiltonian.}

If we assume that $\beta$ and $\alpha$ has the same order of magnitude (M. Andrade; 2003) then the second order Hamiltonian of the problem can written as:

$$
H=H_{0}+H_{\alpha}+H_{\beta}+H_{\alpha \beta}+\mathrm{U}_{\mathrm{R}}
$$

Such that;

$$
\begin{aligned}
& H_{0}=-\frac{\mu_{0}^{2}}{2 Q_{1}^{2}} \\
& H_{\alpha}=\sum_{i=1}^{2} \alpha^{i}\left(A_{i} \tau^{i}+B_{i} \tau^{i-1} \operatorname{Sin} E\right) \\
& H_{\beta}=A_{\beta} \Phi^{2} \sin E \\
& H_{\alpha \beta}=A_{\beta \alpha} \tau \Phi^{2} \sin E \\
& \mathrm{U}_{\mathrm{R}}=\Phi \sum_{i=0}^{2} \alpha^{i} A_{R P . i} \tau^{i}
\end{aligned}
$$

Where;

$$
\begin{aligned}
& \tau=\left(t-t_{0}\right) \\
& \Phi=\frac{a}{r} \\
& A_{1}=\frac{1}{Q_{1}^{2}} \mu_{0}^{n+1} \\
& A_{2}=-\frac{1}{2 Q_{1}^{2}} \mu_{0}^{2 n}(n+1) \\
& B_{1}=-Q_{1} e \mu_{0}^{n-1} \\
& B_{2}=Q_{1} e \mu_{0}^{2 n-2}(n-1)
\end{aligned}
$$




$$
\begin{aligned}
& A_{\beta}=-\beta \frac{Q_{2}}{Q_{1}^{3}} \text { e } \mu_{0} \\
& A_{\beta \alpha}=\beta \alpha \frac{Q_{2}}{Q_{1}^{3}} \text { e } \mu_{0}^{n} \\
& A_{R P .0}=\frac{\mu_{0}}{Q_{1}^{2}} R(\gamma) \\
& A_{R P .1}=-\frac{\mu_{0}^{n}}{Q_{1}^{2}} R(\gamma) \\
& A_{R P .2}=n \mu_{0}^{2 n-1} \frac{1}{2 Q_{1}^{2}} R(\gamma)
\end{aligned}
$$

\section{II- Evaluating D operator.}

To evaluate the $D$ operator, first we will find the variation in the orbital elements using Hamilton's equations of motion.

$q_{\imath}=\frac{\partial H}{\partial Q_{i}}$ and $\dot{Q}_{\imath}=-\frac{\partial H}{\partial q_{i}} \quad i=1,2$

Evaluation of $\dot{q}_{1}$

To calculate $\dot{q}_{1}$ we use Hamiltonian's equation of motion.

Then;

$$
\dot{q}_{1}=\frac{\partial H}{\partial Q_{1}}=\frac{\partial\left[H_{0}+H_{\alpha}+H_{\beta}+H_{\alpha \beta}+\mathrm{U}_{\mathrm{R}}\right]}{\partial Q_{1}}
$$

After calculating the required dervatives in (10) we get:

$$
\dot{q}_{1}=\sum_{j=0}^{2} \Gamma_{j}^{(1)} \Phi^{2 j} \sin E+\sum_{j=1}^{4} \Gamma_{j}^{(2)} \Phi^{j} \sin 2 E+\sum_{j=0}^{1}\left[\Gamma_{j}^{(0)} \Phi^{3} \cos j E+\Gamma_{j}^{(0)} \Phi^{j}\right]
$$

Where;

$$
\begin{array}{lc}
\Gamma_{0}^{(1)}=\sum_{i=1}^{2} \alpha^{i} \tau^{i-1} B_{i ; Q_{1}} & \Gamma_{1}^{(1)}=A_{\beta ; Q_{1}}+\tau A_{\beta \alpha ; Q_{1}} \\
\Gamma_{2}^{(1)}=-2 e\left(A_{\beta}^{\prime}+A_{\beta \alpha}^{\prime} \tau\right) & \Gamma_{2}^{(2)}=0 \\
\Gamma_{1}^{(2)}=\sum_{i=1}^{2} \frac{1}{2} \alpha^{i} \tau^{i-1} B_{i}^{\prime} & \Gamma_{4}^{(2)}=A_{\beta}^{\prime}+\tau A_{\beta \alpha}^{\prime}=2 \Gamma_{3}^{(2)} \\
\Gamma_{3}^{(2)}=\frac{1}{2}\left(A_{\beta}^{\prime}+\tau A_{\beta \alpha}^{\prime}\right) & \Gamma_{1}^{(0)}=\sum_{i=0}^{2} \alpha^{i} \tau^{i} A_{R P . i}^{\prime} \\
\Gamma_{0}^{(0)}=-\sum_{i=0}^{2} \alpha^{i} \tau^{i} e A_{R P . i}^{\prime}=-e \Gamma_{1}^{(0)} & \Gamma_{1}^{(0)}=\sum_{i=0}^{2} \alpha^{i} \tau^{i} A_{R P . i ; Q_{1}} \\
\Gamma_{0}^{(0)}=\frac{\mu_{0}^{2}}{Q_{1}^{3}}+\sum_{i=1}^{2} \alpha^{i} A_{i ; Q_{1}} \tau^{i} &
\end{array}
$$

With ; 


$$
\begin{aligned}
& A_{1 ; Q_{1}}=\frac{-2}{Q_{1}^{3}} \mu_{0}^{n+1} \\
& A_{2 ; Q_{1}}=\frac{1}{Q_{1}^{3}} \mu_{0}^{2 n}(n+1) \\
& B_{1 ; Q_{1}}=-e \mu_{0}^{n-1} \\
& B_{2 ; Q_{1}}=e \mu_{0}^{2 n-2}(n-1) \\
& B_{1}^{\prime}=B_{1} \frac{Q_{2}^{2}}{e Q_{1}^{3}}=-\frac{Q_{2}^{2}}{Q_{1}^{2}} \mu_{0}^{n-1} \quad, \quad B_{2}^{\prime}=B_{2} \frac{Q_{2}^{2}}{e Q_{1}^{3}}=\frac{Q_{2}^{2}}{Q_{1}^{2}} \mu_{0}^{2 n-2}(n-1) \\
& A_{\beta ; Q_{1}}=\beta\left(3 e \frac{Q_{2}}{Q_{1}^{4}} \mu_{0}-\frac{Q_{2}^{3}}{e Q_{1}^{6}} \mu_{0}\right) \\
& A_{\beta}^{\prime}=A_{\beta} \frac{Q_{2}^{2}}{e Q_{1}^{3}}=-\beta \frac{Q_{2}^{3}}{Q_{1}^{6}} \mu_{0} \\
& A_{\beta \alpha ; Q_{1}}=-\beta \alpha\left(3 e \frac{Q_{2}}{Q_{1}^{4}} \mu_{0}^{n}-\frac{Q_{2}^{3}}{e Q_{1}^{6}} \mu_{0}^{n}\right) \\
& A_{\beta \alpha}^{\prime}=A_{\beta \alpha} \frac{Q_{2}^{2}}{e Q_{1}^{3}}=\beta \alpha \frac{Q_{2}^{3}}{Q_{1}^{6}} \mu_{0}^{n} \\
& A^{\prime}{ }_{R P . i}=\frac{Q_{2}^{2}}{e Q_{1}^{3}} A_{R P . i} \quad . i=1,2 \\
& A^{*}{ }_{R P . i}=-\frac{Q_{2}^{2}}{Q_{1}^{3}} A_{R P . i} \\
& A_{R P .0 ; Q_{1}}=-\frac{2}{Q_{1}^{3}} \mu_{0} R(\gamma) \\
& A_{R P .1 ; Q_{1}}=\frac{2}{Q_{1}^{3}} \mu_{0}^{n} R(\gamma) \\
& A_{R P .2 ; Q_{1}}=-n \frac{\mu_{0}^{2 n-1}}{Q_{1}^{3}} R(\gamma)
\end{aligned}
$$

Evaluation of $\dot{q}_{2}$

Again using Hamilton equation of motion to calculate $\dot{q}_{2}$,

$$
\dot{q}_{2}=\frac{\partial H}{\partial Q_{2}}=\frac{\partial\left[H_{0}+H_{\alpha}+H_{\beta}+H_{\alpha \beta}+\mathrm{U}_{\mathrm{R}}\right]}{\partial Q_{2}}
$$

After calculating the required derivatives in (12) we get:

$$
\dot{q}_{2}=\sum_{j=0}^{2} \Pi_{j}^{(1)} \Phi^{2 j} \sin E+\sum_{j=1}^{4} \Pi_{j}^{(2)} \Phi^{j} \sin 2 E+\sum_{j=0}^{1} \Pi_{j}^{(0)} \Phi^{3} \cos j E++\Pi_{0}^{\prime(0)}
$$

Where; 


$$
\begin{array}{lc}
\Pi_{0}^{(1)}=\sum_{i=1}^{2} \alpha^{i} \tau^{i-1} B_{i ; Q_{2}}, & \Pi_{1}^{(1)}=A_{\beta ; Q_{2}}+\tau A_{\beta \alpha ; Q_{2}} \\
\Pi_{2}^{(1)}=-2\left(C_{\beta}-\tau C_{\beta \alpha}\right), & \Pi_{1}^{(2)}=-\sum_{i=1}^{2} \alpha^{i} \tau^{i-1} B_{i}^{\prime \prime}, \quad \Pi_{2}^{(2)}=0 \\
\Pi_{3}^{(2)}=\frac{1}{2}\left(C_{\beta}^{\prime}+\tau C_{\beta \alpha}^{\prime}\right) & \Pi_{4}^{(2)}=C_{\beta}^{\prime}-\tau C_{\beta \alpha}^{\prime} \\
\Pi_{0}^{(0)}=\sum_{i=0}^{2} \alpha^{i} C_{R P, i} & , \quad \Pi_{1}^{(0)}=-\sum_{i=0}^{2} \alpha^{i} A^{\prime \prime}{ }_{R P . i} \\
\Pi_{0}^{\prime(0)}=\sum_{i=1}^{2} \alpha^{i} A_{i ; Q_{2}} \tau^{i} &
\end{array}
$$

With ;

$$
\begin{aligned}
& C_{\beta}=A_{\beta} \frac{Q_{2}}{Q_{1}^{2}}=-\beta \frac{Q_{2}^{2}}{Q_{1}^{5}} e \mu_{0}, C_{\beta \alpha}=A_{\beta \alpha} \frac{Q_{2}}{Q_{1}^{2}}=\beta \alpha \frac{Q_{2}^{2}}{Q_{1}^{5}} e \mu_{0}^{n} \\
& C_{\beta}^{\prime}=A_{\beta} \frac{Q_{2}}{e Q_{1}^{2}}=-\beta \frac{Q_{2}^{2}}{Q_{1}^{5}} \mu_{0}, C_{\beta \alpha}^{\prime}=A_{\beta \alpha} \frac{Q_{2}}{e Q_{1}^{2}}=\beta \alpha \frac{Q_{2}^{2}}{Q_{1}^{5}} \mu_{0}^{n} \\
& A_{R P, 0}^{\prime \prime}=\frac{Q_{2}}{e Q_{1}^{2}} A_{R P, 0}=\frac{\mu_{0} Q_{2}}{e Q_{1}^{4}} R(\gamma), A_{R P, 1}^{\prime \prime}=\frac{Q_{2}}{e Q_{1}^{2}} A_{R P, 1}=-\frac{\mu_{0}^{n} Q_{2}}{e Q_{1}^{4}} R(\gamma) \\
& A_{R P, 2}^{\prime \prime}=\frac{Q_{2}}{e Q_{1}^{2}} A_{R P, 2}=n \mu_{0}^{2 n-1} \frac{Q_{2}}{2 e Q_{1}^{4}} R(\gamma) \\
& C_{R P, i}=e A^{\prime \prime}{ }_{R P . i} \\
& A_{i ; Q_{2}}=0 \quad . i=1.2, B_{1 ; Q_{2}}=\frac{Q_{2}}{e Q_{1}} \mu_{0}^{n-1} \\
& B_{2 ; Q_{2}}=-\frac{Q_{2}}{e Q_{1}} \mu_{0}^{2 n-2}, \quad \\
& B_{1}^{\prime \prime}=B_{1} \frac{Q_{2}}{2 e Q_{1}^{2}}=-\frac{Q_{2}}{2 Q_{1}} \mu_{0}^{n-1}, B_{2}^{\prime \prime}=B_{2} \frac{Q_{2}}{2 e Q_{1}^{2}}=\frac{Q_{2}}{2 Q_{1}} \mu_{0}^{2 n-2}(n-1) \\
& A_{\beta ; Q_{2}}=\beta\left(-\frac{e}{Q_{1}^{3}} \mu_{0}+\frac{Q_{2}^{2}}{e Q_{1}^{5}} \mu_{0}\right), A_{\beta \alpha ; Q_{2}}=\beta \alpha\left(\frac{e}{Q_{1}^{3}} \mu_{0}^{n}-\frac{Q_{2}^{2}}{e Q_{1}^{5}} \mu_{0}^{n}\right) \\
& A_{R P . i} i Q_{2}=0 \quad, i=0,1,2
\end{aligned}
$$

Evaluation of $\dot{Q}_{1}$

Again using Hamilton equation of motion to calculate $\dot{Q}_{1}$,

$$
\dot{Q_{1}}=-\frac{\partial H}{\partial q_{1}}=-\frac{\partial\left[H_{0}+H_{\alpha}+H_{\beta}+H_{\alpha \beta}+\mathrm{U}_{\mathrm{R}}\right]}{\partial q_{1}}
$$

After calculating the required derivatives in (14) we get:

$$
\dot{Q_{1}}=\sum_{i=0}^{1}\left[\Xi_{i}^{(1)} \Phi^{(2 i+1)} \cos E+\Xi_{i}^{(2)} \Phi^{4} \cos 2 E\right]+\sum_{j=0}^{3} \widetilde{\Xi}_{j}^{(2)} \Phi^{j} \sin E+\widetilde{\Xi}_{0}^{\prime(0)}
$$


for $j=1, E$ must equal $\frac{\pi}{2}$

where;

$$
\begin{aligned}
& \Xi_{0}^{(1)}=-\sum_{i=1}^{2} \alpha^{i} \tau^{i-1} B_{i}, \quad \Xi_{1}^{(1)}=-\left(A_{\beta}+\tau A_{\beta \alpha}\right) \\
& \Xi_{0}^{(2)}=-\left(\Psi_{4}+\tau \Psi_{5}\right), \quad \Xi_{1}^{(2)}=0=\widetilde{\Xi}_{1}^{(2)} \\
& \widetilde{\Xi}_{0}^{(2)}=\sum_{i=1}^{2} \alpha^{i} \Psi_{3} \tau^{i-2} \\
& \widetilde{\Xi}_{2}^{(2)}=-\Psi_{6} \quad, \quad \widetilde{\Xi}_{3}^{(2)}=\sum_{i=1}^{2} \alpha^{i} \Psi_{7} \tau^{i} \\
& \widetilde{\Xi}_{0}^{(0)}=\sum_{i=1}^{2} \alpha^{i}\left[\Psi_{i} \tau^{i-1}\right]+\Psi_{4} \Phi^{4}+\Psi_{5} \Phi^{4} \tau+\sum_{i=0}^{2} \alpha^{i}\left[-\Psi_{8+i} \tau^{i-1} \Phi\right]
\end{aligned}
$$

With;

$\Psi_{i}=-i A_{i} \frac{Q_{1}^{3}}{\mu^{2}} \cdot i=1.2 \quad \& \quad \Psi_{3}=-(i-1) B_{i} \frac{Q_{1}^{3}}{\mu^{2}}$

$\Psi_{4}=A_{\beta} e \quad, \quad \Psi_{5}=A_{\beta \alpha} e$

$\Psi_{6}=A_{\beta \alpha} \frac{Q_{1}^{3}}{\mu^{2}} \quad, \Psi_{7}=e A_{R P . i}$

$\Psi_{8+i}=i A_{R P . i} \frac{Q_{1}^{3}}{\mu^{2}}, \quad i=0,1,2$

$A_{R P . ~} i q_{1}=0$. for $i=0,1,2$

Evaluation of $\dot{Q}_{2}$

Again using Hamilton equation of motion to calculate $\dot{Q}_{2}$,

$$
\dot{Q_{2}}=-\frac{\partial H}{\partial q_{2}}=0
$$

\section{III- The first order soluation}

The linear Lie operator $D$, in terms of Delaunay elements, has the general form:

$$
\mathrm{D}=\frac{\mathrm{d} q_{1}}{\mathrm{dt}} \frac{\partial}{\partial q_{1}}+\frac{\mathrm{d} q_{2}}{\mathrm{dt}} \frac{\partial}{\partial q_{2}}+\frac{\mathrm{d} Q_{1}}{\mathrm{dt}} \frac{\partial}{\partial Q_{1}}+\frac{\mathrm{d} Q_{2}}{\mathrm{dt}} \frac{\partial}{\partial Q_{2}}+\frac{\partial}{\partial \mathrm{t}}
$$

Applying the operator $D$ to $q_{1}, q_{2}, Q_{1}, Q_{2}$ and $t$ yields the first order solution for the required variable. Then,

$$
>D q_{1}=\frac{\mathrm{d} q_{1}}{\mathrm{dt}}+\frac{\partial q_{1}}{\partial \mathrm{t}}=\dot{q}_{1}+\tilde{n}
$$

Where $\tilde{n}$ is the mean motion. Using (11) yields:

$$
\vec{q}_{1}\left(q_{1}, q_{2}, Q_{1}, Q_{2}, t\right)=\sum_{j=0}^{1}\left[D^{j} \vec{q}_{1}\right]_{\vec{q}_{1}=\vec{q}_{10}} \frac{\left(t-t_{0}\right)^{j}}{j !}=q_{10}+\left.D q_{1}\right|_{q_{1}=q_{0}} \tau
$$




$$
\begin{aligned}
& =q_{10}+\mid \sum_{j=0}^{2} \Gamma_{j}^{(1)} \Phi^{2 j} \sin E+\sum_{j=1}^{4} \Gamma_{j}^{(2)} \Phi^{j} \sin 2 E+\sum_{j=0}^{1}\left[\Gamma_{j}^{(0)} \Phi^{3} \cos j E+\right. \\
& \left.\Gamma_{j}^{(0)} \Phi^{j}\right]+\left.\tilde{n}\right|_{q_{1}=q_{10}} \tau
\end{aligned}
$$

$$
>D q_{2}=\frac{\mathrm{d} q_{2}}{\mathrm{dt}}=\dot{q}_{1}
$$

Using (13) yields:

$$
\begin{aligned}
& \vec{q}_{2}\left(q_{1}, q_{2}, Q_{1}, Q_{2}, t\right)=\sum_{j=0}^{1}\left[D^{j} \vec{q}_{2}\right]_{\vec{q}_{2}=\vec{q}_{2_{0}}} \frac{\left(t-t_{0}\right)^{j}}{j !} \\
& =q_{20}+\left.D q_{2}\right|_{q_{2}=q_{0}} \tau \\
& =q_{20}+\mid \sum_{j=0}^{2} \Pi_{j}^{(1)} \boldsymbol{\Phi}^{2 j} \sin E+\sum_{j=1}^{4} \Pi_{j}^{(2)} \boldsymbol{\Phi}^{j} \sin 2 E+ \\
& \sum_{j=0}^{1} \Pi_{j}^{(0)} \boldsymbol{\Phi}^{3} \cos E+\left.\Pi_{0}^{\prime(0)}\right|_{q_{2}=q_{20}} \tau \\
& >D Q_{1}=\frac{\mathrm{d} Q_{1}}{\mathrm{dt}}=\dot{q}_{1}
\end{aligned}
$$

$=Q_{10}+\mid \sum_{i=0}^{1}\left[\Xi_{i}^{(1)} \boldsymbol{\Phi}^{(2 i+1)} \cos E+\Xi_{i}^{(2)} \boldsymbol{\Phi}^{4} \cos 2 E\right]+\sum_{j=0}^{3} \widetilde{\Xi}_{j}^{(2)} \boldsymbol{\Phi}^{j} \sin E+$ $\left.\widetilde{\Xi}_{0}^{\prime(0)}\right|_{Q_{1}=Q_{0}} \tau$

$$
\begin{gathered}
>D Q_{2}=\frac{\mathrm{d} Q_{2}}{\mathrm{dt}}=\dot{Q}_{1} \\
\vec{Q}_{2}\left(q_{1}, q_{2}, Q_{1}, Q_{2}, t\right)=\sum_{j=0}^{1}\left[D^{j} \vec{Q}_{2}\right]_{\vec{Q}_{20}} \frac{\left(t-t_{0}\right)^{j}}{j !} \\
=\vec{Q}_{2_{0}}
\end{gathered}
$$

\section{4) Conclusions}

The Hamiltonian function for the motion of spacecraft's around radiated varying mass body was constructed. Perturbation method depending on Lie series and Lie operator was outlined. The algorithm for the sequence of computation was introduced. According to our model, the D operator was derived in terms of Delaunay variables. The solution of the problem, for first order, is obtained. For farther computation, we suggest to apply our model for space craft known its state vector, $\vec{S}_{0}$, at time $t_{0}$ to predict its state vector, $\vec{S}$, at any time t. 


\section{References}

[1] Jeans, J. H. (1924): MNRAS, Vol. 64, No. 1, pp.2-16.

[2] Razbitnaya, E. P. (1985) : Astron. Zh., vol.62, p.1175.

[3] Mestschersky, F. (1949) : Moscow, Gostekhizdat.

[4] Berkovich, L. M. (1980) : Prikl. Mat. Mekh., vol.44, no.2, p.354.

[5] Berkovich, L. M. (1981) : Celest. Mech., vol.24, no.4, p.407.

[6] Salamassi, M. (1985): Celestial Mechanics, 37, 359-369.

[7] Polyakhova, E. N. (1994): Astron. Rep., Vol. 38, No. 2, pp. 283-291.

[8] Prieto, C. (1995): Publications of the Department of applied Mathematics, University de Santiago, de Compostela - Spain.

[9] Hadjidemetriou, J. D. (1963) : Icarus, vol.2, p.440.

[10] Hadjidemetriou, J. D. (1966) : Icarus, vol.5, p.34.

[11] Van Der Laan. L. y and Verhulst, F. (1972) : Celest. Mech., p. 6, pp. 343-351.

[12] Verhulst, F.:(1969). Bull. Astron. Inst. Neth., 20, 215-221.

[13] Verhulst, F.:(1969). Bull. 5th ICNO Conference, Kiev, 158-168.

[14] Verhulst, F. y and Eckhaus, W.:(1970). Int. J. Non-Linear Mechanics, 5, 617-624.

[15] Jeans, J. H. (1925): MNRAS, Vol. 85, No. 9, pp. $912-925$.

[16] Prieto, C. and Docobo, J. A.(1997 a): Astron. Astrophys., Vol. 318, pp. 657-661.

[17] Prieto, C. and Docobo, J. A. (1997b): Celes. Mech. \&Dyn. Astron., Vol. 68, pp. 53.

[18] Docobo, J. A., Blanco, J. and Abelleira, P. (1999): Monografias de la Academiade Ciencias de Zaragoza, 14, II Jornadas de Mecanica Celeste, eds. A. Elipe, \& V. Lanchares (Zaragoza: Academia de Ciencias de Zaragoza), 33 (in Spanish).

[19] [46] Andrade, M. and Docobo J. A. Detecting variations of the orbital elements due to periastron effect in eclipsing binaries., Astronomical Observatory R.M. Aller Universidade de Santiago de Compostela 15782 Santiago de Compostela, Galiza (Spain) Monograf'ıas de la Real Academia de Ciencias de Zaragoza 35, 112, (2011).

[20] Andrade, M., em Métodos de dinámica orbitaly rotacional: IV Jornadas de Trabajo en Mecánica Celeste, eds. S. Ferrer, T. López\& A. Vigueras (Murcia: Prensas Universitarias), 2002, p. 113-120 (in Spanish), (2002).

[21] Andrade, M. \& Docobo, J. A. Estudio de la estabilidad en sistemas estelares triples conp'erdida de masa. Mon. Acad. Cienc. Zaragoza, 25, 13-22, (2004).

[22] Rahoma, W. A. et al. (2009): J. Astrophys. and Astr., Vol 30, pp. 187-205. 
[23] Delva, M. (1984) : Celest. Mech., vol.34, p.145.

[24] HalsImeier, A. (1984) : Celest. Mech., vol.3, p.107.

[25] El-Saftawy, M. I. and Algethami, A. R. (2014): International Journal of Astronomy and Astrophysics, Vol. 4, pp. 70-79.

[26] Deprit, A. (1983): Celest. Mech., Vol. 31, pp. 1-22G. Eason, B. Noble, and I. N. Sneddon, "On certain integrals of Lipschitz-Hankel type involving products of Bessel functions," Phil. Trans. Roy. Soc. London, vol. A247, pp. 529-551, April 1955. (references)

[27] J. Clerk Maxwell, A Treatise on Electricity and Magnetism, 3rd ed., vol. 2. Oxford: Clarendon, 1892, pp.6873.

[28] I. S. Jacobs and C. P. Bean, "Fine particles, thin films and exchange anisotropy," in Magnetism, vol. III, G. T. Rado and H. Suhl, Eds. New York: Academic, 1963, pp. 271-350.

[29] K. Elissa, "Title of paper if known," unpublished.

[30] R. Nicole, "Title of paper with only first word capitalized," J. Name Stand. Abbrev., in press.

[31] Y. Yorozu, M. Hirano, K. Oka, and Y. Tagawa, "Electron spectroscopy studies on magneto-optical media and plastic substrate interface," IEEE Transl. J. Magn. Japan, vol. 2, pp. 740-741, August 1987 [Digests 9th Annual Conf. Magnetics Japan, p. 301, 1982].

[32] M. Young, The Technical Writer's Handbook. Mill Valley, CA: University Science, 1989. 